\title{
New extension of the Fontan principle: inferior vena cava-pulmonary artery bridge operation
}

\author{
SUGATO NAWA, SHIGERU TERAMOTO \\ From the Second Department of Surgery, Okayama University Medical School, Ukayama, Japan
}

\begin{abstract}
A new modification of the Fontan operation for complex cardiac anomalies is described. The technique comprises superior vena cava-pulmonary artery anastomosis and inferior vena cava-pulmonary artery bridging via a composite conduit connected to the inferior vena cava via the right atrium, totally excluding the right heart. The procedure was applied to a patient with complex cardiac abnormalities associated with asplenia. After operation the patient had good haemodynamic function initially though he died from multiorgan failure after airway bleeding on the 24th postoperative day. The procedure is a simple alternative to the complicated atrial partition required for the conventional Fontan operation.
\end{abstract}

\section{Introduction}

The conventional Fontan principle introduced for patients with a hypoplastic or absent right ventricle depends on some form of anastomosis between the right atrium and pulmonary artery. Although the results of such operations are now excellent,' the results of the modified Fontan operation ${ }^{2}$ for complex cardiac anomalies are not yet satisfactory, particularly in cases requiring concomitant intra-atrial partition. ${ }^{3}$ The technique used in this case is a possible alternative to the problematic intra-atrial baffle operations, and may improve the results when the Fontan operation is modified in this way.

\section{Case report}

A 6 year old boy was admitted for re-evaluation of complex cardiac anomalies associated with the asplenia syndrome. Echocardiography and cardiac catheterisation showed the following anomalies: common atrium, common ventricle (type C), complete atrioventricular canal with a common atrioventricular valve (type $\mathrm{C}$ ), transposed great arteries (situs ambiguous, d-loop, l-transposition), pulmonary stenosis, bilateral venae cavae draining into each side of a common atrium, the left sided inferior vena cava opening into the right side of the atrium, absence of the coronary sinus and the right aortic arch. The pressure in the common atrium (a and $v$ waves) was $15 / 13$ (mean 14) $\mathrm{mm} \mathrm{Hg}$, in the

Address for reprint requests: Dr S Nawa, Okayama University Medical School, 2-5-1 Shikata-cho, Okayama, 700 Japan.

Accepted 19 May 1988 pulmonary trunk (systolic (S)/diastolic (D)) 20/14 (mean 17) $\mathrm{mm} \mathrm{Hg}$, in the aorta (S/D) 83/62 (mean 68) mm Hg. Trivial regurgitation of a common atrioventricular valve was shown at ventriculography. The right and left pulmonary arteries were normal, though pulmonary stenosis was seen below, at, and above the valve. No subaortic stenosis was noted. This patient was operated on by a new modification of the Fontan procedure on 14 July 1986.

\section{OPERATIVE TECHNIQUES}

A composite conduit was made at the time of surgery with two kinds of material. One was an externally, spirally stented polytetrafluoroethylene graft (IMPRA-FLEX graft, Impra Inc, Arizona USA) and the other was a woven Dacron vascular graft (Meadox woven double velour, Meadog Medicals Inc, Ockland, New Jersey, USA). The formew (19 mm diameter) was partially intussuscepted into the latter (22 $\mathrm{mm}$ diameter) and the intussuscepted end was ans stomosed to the Dacron graft half way along its length (figure).

End to side anastomosis of the superior vena cava with the pulmonary artery was done on each side one by one as reported by Kawashima et $a l^{4}$ and Vargas et al. ${ }^{5}$ After this cardiopulmonary bypass was instituted, aortic root perfusion, direct cannulation in each superior vena cava, and drainage from the inferior vena cava via the right femoral vein being used. A cross clamp was placed on the intrapericardial inferior vena cava, and the right atrium was incised. The aorta was cross clamped and cardioplegia given with the St Thomas's solution. A large common atrioventricular valve was inspected and its competency preserved without surgical correction. The Dacron graft was cut off, leaving about $5 \mathrm{~mm}$ beyond its connection to the IMPRAFLEX graft, and the end was anastomosed with the inferior vena cava inside the atrium at the cavoatrial junction. The other end of the Dacron graft was also cut off where the graft came out through the atrial incision, and was circumferentially sutured to the incised atrial wall. Then the end of the IMPRA-FLEX graft was connected to the side of the pulmonary trunk, and the pulmonary root was doubly ligated (figure).

The patient was weaned from the cardiopulmonary bypass with a minimal dose of inotropes and circulatory volume expansion as in the conventional Fontan operation. Postoperatively the mean pressure was $17,18,18,17,17,18$, and $8 \mathrm{~mm} \mathrm{Hg}$ in the right and left superior vena cava, the inferior vena cava, the right and left and main pulmonary arteries, and the atrium (functional left atrium) respectively. The systolic and diastolic ventricular pressure was measured as 


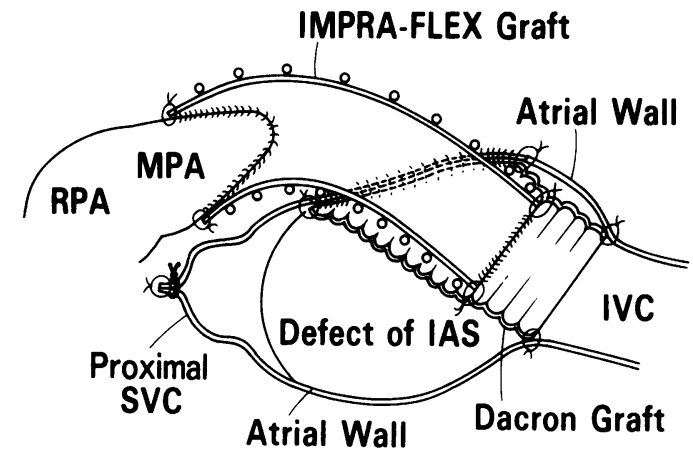

Schematic illustration of the procedure. A sheathed composite conduit can bridge the inferior vena cava (IVC) to the pulmonary artery, totally excluding the right heart. MPAmain pulmonary artery; $R P A$-right pulmonary artery; $S V C$-superior vena cava; IVC-inferior vena cava; IASinteratrial septum.

$115 / 8$ (mean 55 ) $\mathrm{mm} \mathrm{Hg}$ and the aortic pressure as $120 / 90$ (mean 115) $\mathrm{mm} \mathrm{Hg}$. The patient had recovered sufficiently to take food by mouth on the eighth postoperative day, and he had advanced to intermittent mandatory ventilation. After unexpected airway bleeding, however, multiorgan failure developed, with hepatic failure, gastrointestinal bleeding, and finally renal failure. The patient died on the 24th postoperative day. The circulation, however, remained stable to the end. Consent for necropsy was not given.

\section{Discussion}

The contribution of the right atrial contraction to the Fontan circulation is controversial. Using pulsed Doppler echocardiography, we have observed that the pulmonary blood flow pattern and velocity depend on the right atrial contraction in a classical Fontan circulation. Moreover, many papers reported that the onset of atrial fibrillation led to deterioration of the Fontan circulation. We do not deny that there is an atrial contribution, and it may to some extent assist the circulation. Nevertheless, we think that the essential haemodynamic source of the Fontan circulation is derived not from the atrial contraction but from vis a tergo- that is, the left ventricular pump function. Matsuda et $a^{6}$ have proved experimentally that atrial contraction is not essential for the Fontan circulation, and the case of Kawashima et al ${ }^{4}$ in which the right heart was totally excluded provides clinical confirmation. In the reported cases, ${ }^{4}$ however, only the fortuitous circumstance that the inferior vena cava system was anatomically connected to the superior vena cava system enabled total cavopulmonary diversion to be achieved. Otherwise an intra-atrial baffle operation would have been necessary. Di Carlo et al have described the limitations of the Fontan procedure with the atrial partition. ${ }^{2}$ Intra-atrial septation is complicated and has a high risk of problems, such as surgical block, residual shunt, and intra-atrial obstruction. In the reported case the intra-atrial septation technique might have been performed as there was a common atrium, not atrial isomerism. To avoid the possible risks of the aforementioned complications with the intra-atrial partition, however, we applied the new procedure, which we found very simple, easy, and reliable.

In corrective cardiac surgery the use of a large amount of foreign material (for example, in the conduit) should be minimised to eliminate problems arising from the materials used.' The use of prosthetic materials, however, extends the application of the operative principle and many patients will benefit. We have had an excellent result with polytetrafluoroethylene in reconstructing the venous system ${ }^{8}$ and have reported its usefulness in the Fontan operation. ${ }^{8}$ Neither thrombus formation nor development of intraluminal peel has been observed in our series, in which the longest follow up period is 10 years. An external Dacron graft was used not only to make it easy to suture a rigid IMPRA-FLEX graft to the inferior vena cava and the incised atrial wall but also to prevent thrombus formation on the stented, uneven outer surface of the IMPRA-FLEX graft, which was partially placed in the pulmonary venous atrium after this procedure. The Dacron graft used as a conduit was restricted to a very short part less than $5 \mathrm{~mm}$ in length, as it had a high risk of thrombus formation or obstruction, or both, particularly when it was used in the venous system. ${ }^{7}$ The outer surface of the Dacron graft will be endotheliarised in the same way as a patch used for closure of a ventricular septal defect.

Recently, Vargas et al reported a similar technique, ${ }^{3}$ in which a tube graft was placed in the pulmonary venous chamber. The material that was used was not stated, but we believe that contact between arterial blood and the outer surface of the tube graft should be minimised.

Unfortunately our patient died. The cause was neither impairment of the pulmonary circulation or failure to sustain the Fontan circulation, since the circulation was sufficiently sustained even when multiorgan failure ensued. Our technique provides total exclusion of the right heart whether or not the inferior vena cava system is connected to the superior vena cava system, as is needed for a cavopulmonary shunt operation. ${ }^{4}$ We recommend our modification of the Fontan operation for patients requiring problematic intra-arterial partition, particularly those with atrial isomerism. Each patient must be carefully evaluated, however, and the long term outcome of such a conduit in terms of patency remains to be elucidated.

\section{References}

1 Sanders SP, Wright GB, Keane JF, Norwood WI, Castaneda AR. Clinical and hemodynamic results of the Fontan operation for tricuspid atresia. Am J Cardiol 1982;49:1733-40.

2 Fontan F, Baudet R. Surgical repair of tricuspid atresia. Thorax 1971;26:240-8.

3 Di Carlo D, Marcelletti C, Nijveld A, Lubbers LJ, Becker AE. The Fontan procedure in the absence of the interatrial septum: failure of its principle? J Thorac Cardiovasc Surg 1983;85: 923-7.

4 Kawashima Y, Kitamura S, Matsuda H, Shimazaki Y, Nakano S, Hirose H. Total cavo-pulmonary shunt operation in complex cardiac anomalies. A new operation. J Thorac Cardiovasc Surg 1984;87:74-81.

5 Vargas FJ, Mayer JE, Jonas RA, Castaneda AR. Anomalous systemic and pulmonary venous connections in conjunction with atrio-pulmonary anastomosis (Fontan-Kreutzer). Technical considerations. J Thorac Cardiovasc Surg 1987;93: 523-32.

6 Matsuda H, Kawashima Y, Takano H, Miyamoto K, Mori T. Experimental evaluation of atrial function in right atriumpulmonary artery conduit operation for tricuspid atresia. $J$ Thorac Cardiovasc Surg 1981;81:726-7.

7 Ben-Shachar G, Nicoloff DM, Edwards JE. Separation of neointima from Dacron graft causing obstruction. Case following Fontan procedure for tricuspid atresia. J Thorac Cardiovasc Surg 1981;82:268-71.

8 Nawa S, Matsuki T, Shimizu A, et al. Pulmonary artery connection in the Fontan procedure. Flexible polytetrafluoroethylene conduit for expansion. Chest 1987; 91:552-7. 